\title{
The effect of Staphylococcus epidermidis cells on Pseudomonas aeruginosa-associated virulence factors
}

\author{
Suhaga Dohare ${ }^{1}$, Devendra Singh ${ }^{1}$, Deepmala Sharma ${ }^{2}$, Vishnu Agarwal ${ }^{1 *}$ \\ ${ }^{1}$ Department of Biotechnology, Motilal Nehru National Institute of Technology, Allahabad, India. \\ ${ }^{2}$ Department of Mathematics, National Institute of Technology, Raipur, India.
}

\section{ARTICLE INFO \\ Article history: \\ Received on: March 20, 2021 \\ Accepted on: May 21, 2021 \\ Available online: November 10, 2021}

Key words:

Staphylococcus epidermidis,

Pseudomonas aeruginosa,

virulence factors, quorum-sensing,

Escherichia coli $\mathrm{pJN105LpSC11}$

\begin{abstract}
Staphylococcus epidermidis and Pseudomonas aeruginosa are the two most commonly detected bacteria. Pseudomonas aeruginosa exerts significant impacts on pathogenesis through quorum-sensing regulation, while $S$. epidermidis role has always remained a matter of interest due to its frequent presence in most of the hospitalized samples and even inside the body during operation procedures. In this study, the effect of $S$. epidermidis cells on $P$. aeruginosa pathogenesis was analyzed. The effect of $S$. epidermidis cells on $P$. aeruginosa growth was analyzed by using microtiter plate assay, Colony forming unit (CFU), and microscopy. The effect of virulence factors including protease, rhamnolipid, and swarming motility was also investigated. Escherichia coli $\mathrm{pJN105LpSC11,} \mathrm{a} \mathrm{bioreporter} \mathrm{strain,} \mathrm{was} \mathrm{used} \mathrm{to} \mathrm{analyze} \mathrm{the} \mathrm{effect} \mathrm{of} S$. epidermidis on $P$. aeruginosa quorum-sensing. The growth of $P$. aeruginosa did not affect $S$. epidermidis cells of $5.5 \times 10^{9}, 6 \times 10^{9}$, $7 \times 10^{9}, 8.5 \times 10^{9}$, and $1.5 \times 10^{10} \mathrm{CFU} / \mathrm{ml}$, and microscopy results are consistent with the findings. $P$. aeruginosaassociated virulence factors show that with increasing $S$. epidermidis counts, $P$. aeruginosa-associated virulence factors were reduced. No effect of $S$. epidermidis on $P$. aeruginosa quorum-sensing was observed. The outcomes suggest that $S$. epidermidis can be used as an alternative to reduce Pseudomonas aeruginosaassociated virulence factors and its pathogenesis.
\end{abstract}

\section{INTRODUCTION}

Naturally, bacterial species coexist in a multicellular community. Staphylococcus epidermidis is a typical bacterium of human skin flora that shows recurrent presence inside the human and has been frequently reported with Pseudomonas aeruginosa in infections [1]. These two species are repeatedly found in the urinary tract, medical devices, and hydrogel ocular lenses. In a mixed community, $P$. aeruginosa is identified as the most virulent pathogen in comparison with other bacteria and fungi. Pseudomonas aeruginosa secretes quorum-sensing (QS)regulated virulence factors. QS is a microbial communication process in which microbes sense threshold cell density and start secreting QS molecules. Gram-negative class of bacteria has an acyl-homoserine lactone (AHL)-based QS-signaling system. Pseudomonas aeruginosa has two major AHL-based QS systems,

*Corresponding Author

Vishnu Agarwal, Department of Biotechnology, Motilal Nehru National Institute of Technology, Allahabad, India.E-mail:vishnua@mnnit.ac.in including lasI/R and rhlI/R, involving ( $N$-(butanoyl)-L-homoserine lactone) $\left(\mathrm{C}_{4}-\mathrm{HSL}\right)$ and $3-\mathrm{O}-\mathrm{C}_{12}-\mathrm{HSL}$ ( $\mathrm{N}$-(3-oxododecanoyl)-Lhomoserine lactone) molecules, respectively [2].

These QS systems regulate various virulence factor expressions, including proteases, rhamnolipids, pyocyanin extracellular polysaccharides, and swarming [3]. The virulence factors play a vital role in the survival, colonization of the bacteria inside the host, the invasion of tissues, and provide protection against the human immune system. Pseudomonas aeruginosa-associated virulence factors such as pyocyanin, rhamnolipid, extracellular polysaccharide, and Las A protease exert anti-staphylococcal activity [4].

Pseudomonas aeruginosa and its associated factors continue their efforts to lead over other bacterial species, such as S. epidermidis [4]. Recently, it was shown that the viability of Bacillus subtilis, Burkholderia cepacia, Escherichia coli, Stenotrophomonas maltophilia, Staphylococcus aureus, and Candida albicans was impaired in the presence of $P$. aeruginosa in mixed culture by secretion of virulence factors such as pyocyanin, proteases, and 
alginate [5-8]. A microbial species can modulate the virulence of other microbes. The role of $S$. epidermidis in $P$. aeruginosaassociated virulence factors secretion has not been studied so far. In this study, we investigated how $S$. epidermidis alters virulence factor secretions from $P$. aeruginosa. This study will provide more insight into the interaction between two bacterial species. The interactions therein will be essential to design effective therapeutic strategies to combat multispecies infection.

\section{MATERIALS AND METHODS}

\subsection{Bacterial Strains and Growth}

Staphylococcus epidermidis 435 and $P$. aeruginosa PAO1 strains were procured from the MTCC IMTECH, Chandigarh. Bioreporter strain E. coli pJN105L pSC11 was a generous gift by Dr. Peter Greenberg (University of Washington). Azocasein, trichloroacetic acid (TCA), gentamycin, ampicillin, and $\mathrm{C}_{12}$-HSL (3-oxo- $\mathrm{C}_{12}$ homoserine lactone) were procured from Sigma-Aldrich, India. Crystal violet (CV) agar media and Pseudomonas isolation agar media were purchased from Himedia, India. Pseudomonas aeruginosa was cultivated in Luria-Bertani (LB; Himedia, India) medium, $S$. epidermidis strain was cultivated in Tryptic Soy Broth (Himedia, India) and maintained at $37^{\circ} \mathrm{C}$ for 24 hours.

\subsection{Preparation of Single and Mixed Cultures}

Different concentrations of $S$. epidermidis with a fixed concentration of $P$. aeruginosa in mixed culture was prepared [9]. Overnight-grown cultures of $S$. epidermidis and $P$. aeruginosa were centrifuged. The pellets were rinsed with (phosphate buffer saline, $\mathrm{pH}$ 7.4), and desired $\mathrm{OD}_{600}$ was set as per experimental requirements, and the corresponding $\mathrm{CFU} / \mathrm{ml}$ was counted using selective media. To analyze the impact of different cell numbers of $S$. epidermidis on $P$. aeruginosa-associated virulence factors, different numbers of $S$. epidermidis cells with $0.5,0.6,0.7,0.8$ and $0.9 \mathrm{OD}_{600}$, corresponding to $5.5 \times 10^{9}, 6 \times 10^{9}, 7 \times 10^{9}, 8.5 \times 10^{9}$, and $1.5 \times 10^{10} \mathrm{CFU} / \mathrm{ml}$, respectively, was added to $0.5 \mathrm{OD}_{600}$ culture of $P$. aeruginosa corresponding to $5.5 \times 10^{9}(\mathrm{CFU} / \mathrm{ml})$, and these mixed cultures were termed as M1, M2, M3, M4, M5 (M1-M5), respectively. LB broth medium was used for the preparation of single and mixed cultures.

\subsection{Effect of $S$. epidermidis Cells Concentration on the P. aeruginosa Growth}

Pseudomonas aeruginosa growth in single and mixed cultures was studied using microtiter plate assay. Briefly, mixed cultures ranging from M1 to M5 were added to a 96-well polystyrene flatbottom plate. The microtiter plate was then kept for 24 hours at $37^{\circ} \mathrm{C}(100 \mathrm{rpm})$ in a shaking incubator. At different time intervals $\left(2,4,6,8,10\right.$, and 24 hours), $\mathrm{OD}_{600}$ was recorded and the growth curve was plotted [8].

\subsection{Microscopic Study}

\subsubsection{Light microscopy}

For microscopic analysis, PAO1 and S. epidermidis alone and PAO1 along with SE in mixed cultures were grown in LB broth and incubated for 24 hours at $37^{\circ} \mathrm{C}$. After incubation, $20 \mu \mathrm{l}$ of
PAO1 and S. epidermidis alone, along with mixed culture, was visualized under the light microscope at $100 \times$ after Gram-staining.

\subsubsection{Scanning electron microscope (SEM) and viability of co-culture}

Briefly, mixed cultures were in LB broth and incubated for 24 hours at $37^{\circ} \mathrm{C}$. After 24 hours incubation, cell suspensions were centrifuged at 10,000 rpm for 10 minutes, and the pellets washed thrice with $0.1 \mathrm{M}$ phosphate buffer ( $\mathrm{pH} 7.4$ ). Then, they were fixed with $4 \%$ paraformaldehyde and $2.5 \%$ glutaraldehyde $(0.1 \mathrm{M}$ phosphate buffer). The fixative was discarded after centrifugation and the pellets were dehydrated with $10 \%, 30 \%, 50 \%, 70 \%$, and $100 \%$ ethanol for 30 minutes and kept for drying. After the completion of drying, the samples were coated with goldpalladium and observed under SEM [10].

\subsection{Estimation of Virulence Factors}

\subsubsection{Pyocyanin extraction}

Pyocyanin extraction was carried out according to the described methods with some modifications [5]. Briefly, the supernatant of control and mixed cultures was mixed with chloroform, followed by hydrogen chloride. The pink layer obtained after the addition of $\mathrm{HCl}$ was taken, and absorbance was taken at $520 \mathrm{~nm}$ [6].

\subsubsection{Azocasein assay}

Azocasein assay was used to estimate the protease production by $P$. aeruginosa alone and in mixed culture. Azocasein was added to the culture supernatant and incubated for 1 hour. TCA was used to stop the reaction, followed by centrifugation [6]. The supernatant was collected and mixed with one molar sodium hydroxide. The protease activity was then measured by a spectrophotometer at $440 \mathrm{~nm}[11]$.

\subsubsection{Alginate production}

The CV glycolipid method was used to quantify alginate. Calcium chloride was added to the culture supernatant of all mixed and control sets of experiments, followed by centrifugation to obtain a pellet of alginate. The pellet was then stained with $\mathrm{CV}$. The extra stain was removed and acetic acid was used to extract the absorbing dye. The OD was taken at $600 \mathrm{~nm}$ [12].

\subsubsection{Rhamnolipid production}

Rhamnolipid production was determined in the $P$. aeruginosa alone and mixed culture by Siegmund and Wagner [13] agar plate method. The cultures were grown in LB broth for 24 hours, and then agar plates were prepared. Then, inoculums of single and mixed cultures were loaded into wells. The plates were kept in an incubator for 48 hours. The diameter of the blue halo formed around each well was measured.

\subsubsection{Swarming assay}

Swarming motility was assessed on agar plates containing $1 \%$ glucose, $0.5 \%$ peptone, $0.5 \%$ bactoagar, and $0.2 \%$ yeast extract. Exactly $5 \mu \mathrm{l}$ of the single and mixed culture were spotted in the middle of the swarming agar plate and kept for 24 hours at $37^{\circ} \mathrm{C}$. 
The swarming motility was determined by the length and direction of the tendrils formed $[6,14]$.

\subsection{Autoinducer Bioassay}

Escherichia coli pJN105L pSC11 was used as a bioreporter strain to quantify $\mathrm{QS}$ molecule $\left(\mathrm{C}_{12}-\mathrm{HSL}\right)$ produced by $P$. aeruginosa and diluted $E$. coli pJN105LpSC11 was grown in the presence of sterilized culture supernatant of all mixed cultures along with control (1:1) and was incubated for 2 hours. After incubation, 1 $\mathrm{ml}$ cells was diluted in Z-buffer ( $\mathrm{pH} 7.0,1: 1)$, and $\beta$-galactosidase activity was measured by using Miller assay [15].

\subsection{Statistical Analysis}

The analysis was carried out in triplicate. The acquired outcomes are presented as average and standard deviation. All statistical analyses were calculated by the Prism5 software. One-way analysis of variance and Dunnett's multiple comparison test was used for comparison, and the $p$-value less than 0.05 was considered significant.

\section{RESULTS AND DISCUSSION}

\subsection{Co-Survival Studies}

The primary focus of this research work was to assess the growth of $P$. aeruginosa in mixed cultures. Figure 1 shows the SEM images of M1 mixed culture at different time intervals ranging from 2 to 24 hours. In all time frames of M1 mixed culture, the number of $P$. aeruginosa cells was higher than $S$. epidermidis. In the M1 mixed culture, initially, the number of $S$. epidermidis cells was increased in a time-dependent manner from 2 to 6 hours. However, after 8 hours onward, the cell numbers decreased, up to 24 hours. At 24 hours, no or negligible growth of $S$. epidermidis was found when compared to $P$. aeruginosa.

On the contrary, cells of $P$. aeruginosa increased with time, indicating that there is no inhibitory effect of $S$. epidermidis over $P$. aeruginosa cells in mixed culture. Furthermore, the CFU was analyzed after 24 hours of growth. When single species and mixed species culture (M1) were compared, it was observed that $S$. epidermidis was negatively affected in mixed cultures, and the occurrence of $P$. aeruginosa was comparatively higher than $S$. epidermidis (Figure 2).

\subsection{Effect of $S$. epidermidis Cells Concentration on the Growth of $P$. aeruginosa}

Next, the growth curve analysis was carried out in mixed cultures from M1 to M5. The increased growth rate was observed in the case of $S$. epidermidis alone than that of $P$. aeruginosa alone. M1M3 showed growth similar to $P$. aeruginosa alone, but in M4-M5 upward, a shift in the growth curve was observed (Figure 3). A significant decline in the growth of $S$. epidermidis was observed in co-culture and showed lesser persistence than $P$. aeruginosa. On the other hand, at different time periods, the growth of $P$. aeruginosa was not affected in the presence of $S$. epidermidis. The results reveal that the $P$. aeruginosa outcompeted $S$. epidermidis [7].

Further study was continued in the M1-M5 mixed culture. The data depicted that the growth rate was not affected in M1-M3
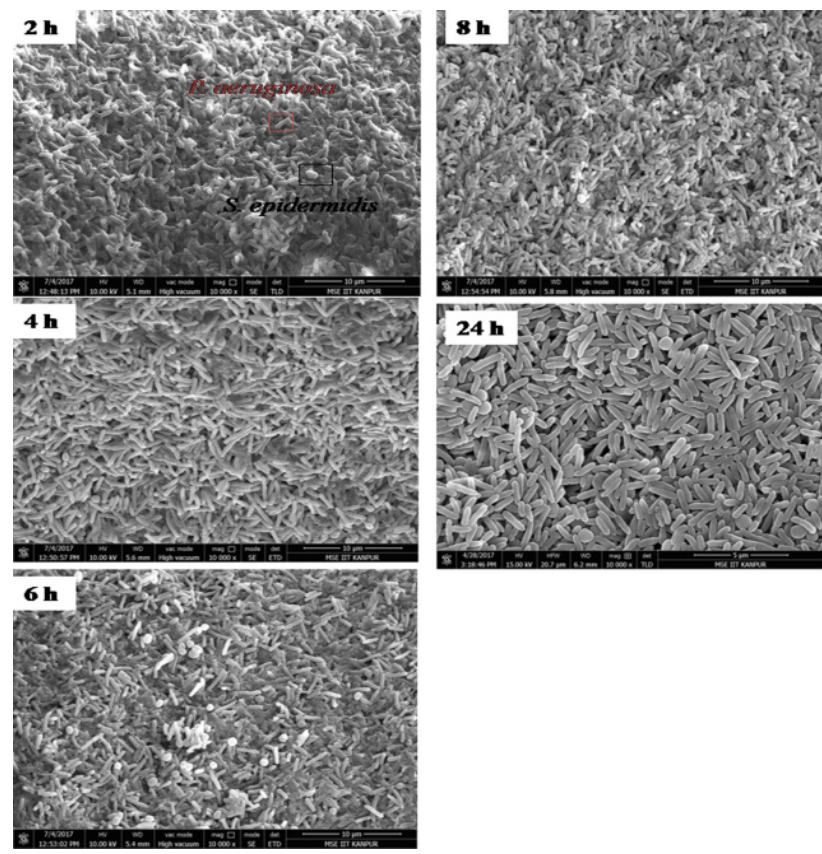

Figure 1: Scanning electron microscopy images of M1 mixed culture at different time intervals (2-24 hours) with scale bars $(3 \mu \mathrm{m})$.

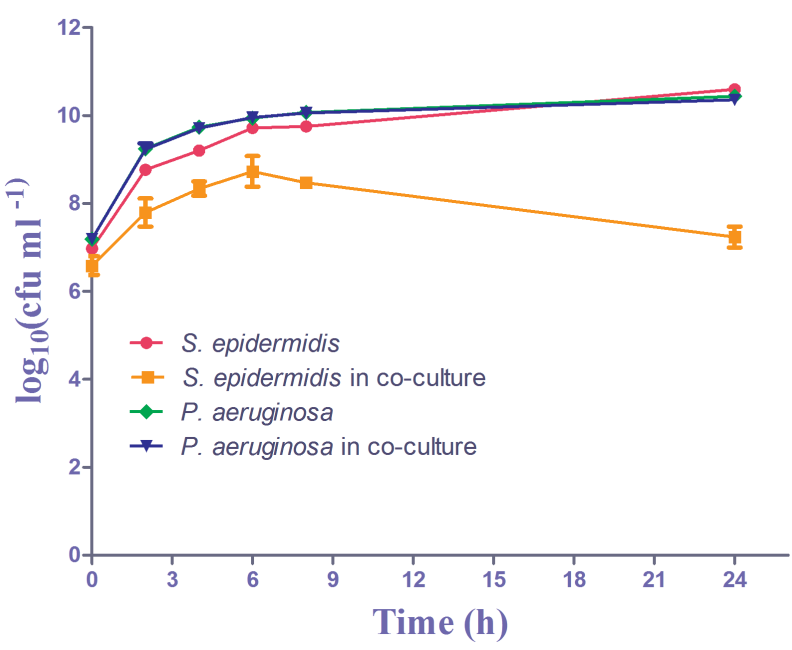

Figure 2: Viability of M1 co-culture at different time intervals $(0,2,4$, 6,8 , and 24 hours). Error bars indicate the \pm standard deviations of the measurements.

mixed culture and was the same as in the case of $P$. aeruginosa. However, an elevated growth curve in the M4-M5 mixed culture was found due to the increased co-survivability of $S$. epidermidis.

\subsection{Microscopic Study}

For confirmation, the microscopic analysis (Gram-staining and SEM) was carried out to investigate the survivability of $S$. epidermidis after 24 hours growth in an M1-M5 mix. Figure 4A and $\mathrm{B}$ shows the microscopic images of $P$. aeruginosa alone and Figure $4 \mathrm{C}$ and D shows the images of $S$. epidermidis alone. Light microscopy and SEM data depicted that in the M1-M3 mix, $P$. aeruginosa was dominated, while only a few cells of $S$. epidermidis 


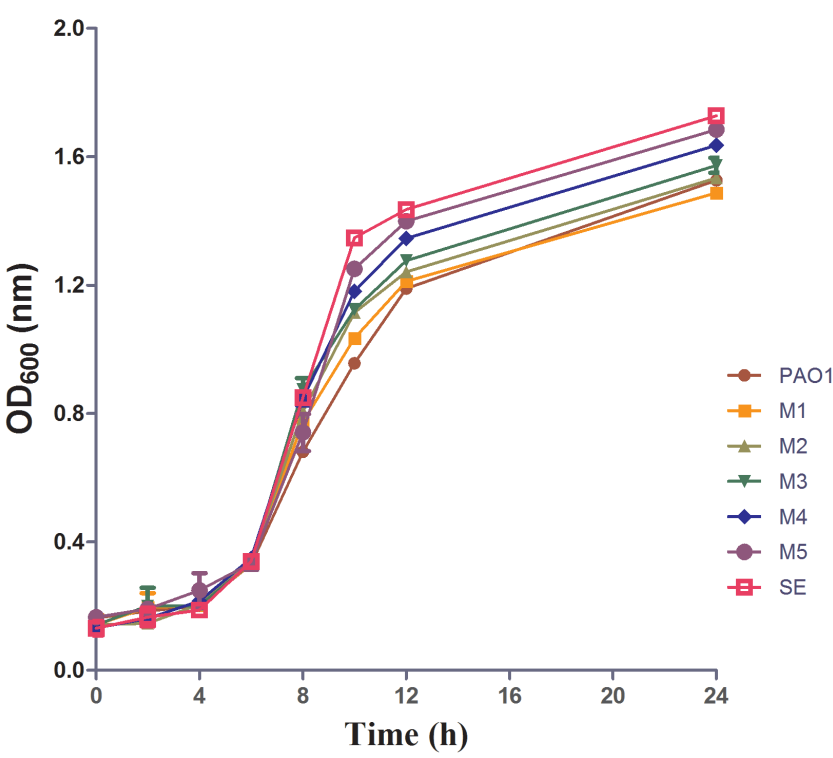

Figure 3: Growth patterns of $P$. aeruginosa (PAO1) and S. epidermidis (SE) in single and mixed (M1-M5) cultures. Error bars indicate the \pm standard deviations of the measurements.

were observed (Figure 4E-J). In the case of M4 and M5 mixed cultures, the significant presence of $S$. epidermidis and sporadic presence of $P$. aeruginosa was observed (Figure $4 \mathrm{~K}-\mathrm{N}$ ); thus, further studies were carried out by taking the M1-M3 mixed culture only. Microscopic analysis revealed the trivial presence of S. epidermidis in the M1-M3 mixed and significant presence in the case of M4 M5 mixed. But co-survivability of $P$. aeruginosa was affected in M4-M5 mixed culture [14]. The present study also suggested that the antagonistic effect of $P$. aeruginosa on $S$. epidermidis, as well as $P$. aeruginosa, outcompeted $S$. epidermidis in planktonic conditions.

\subsection{Effect of $S$. epidermidis on $P$. aeruginosa-Associated Virulence Factors}

Pseudomonas aeruginosa dominates over other species, including $S$. epidermidis, through the secretion of various factors and QS molecules. The secretion of virulence factors by $P$. aeruginosa can be affected by environmental conditions. Thus, the production of $P$. aeruginosa-associated virulence factors in the presence of $S$. epidermidis was investigated against different cell numbers from mixed cultures (M1-M3). Since $P$. aeruginosa-associated virulence factors depend upon its growth, Figures 3 and $4 \mathrm{~K}-\mathrm{N}$ show that the growth of $P$. aeruginosa was negatively affected in M4 and M5. Therefore further studies for evaluation of virulence factors were carried out by taking the M1-M3 mixed cultures only.

\subsubsection{Pyocyanin production}

Pseudomonas aeruginosa secretes many virulence factors that help them dominate over other species, like Pyocyanin, that exert an antibiotic effect, helping to persist in the same environment. Pyocyanin is an extracellular secondary metabolite and harbors antibiotic activity. In the current study, pyocyanin production was assessed, and it was found that in comparison to PAO1 alone, pyocyanin production was decreased from M1 to M3 (Figure 5A) mixed cultures [7].

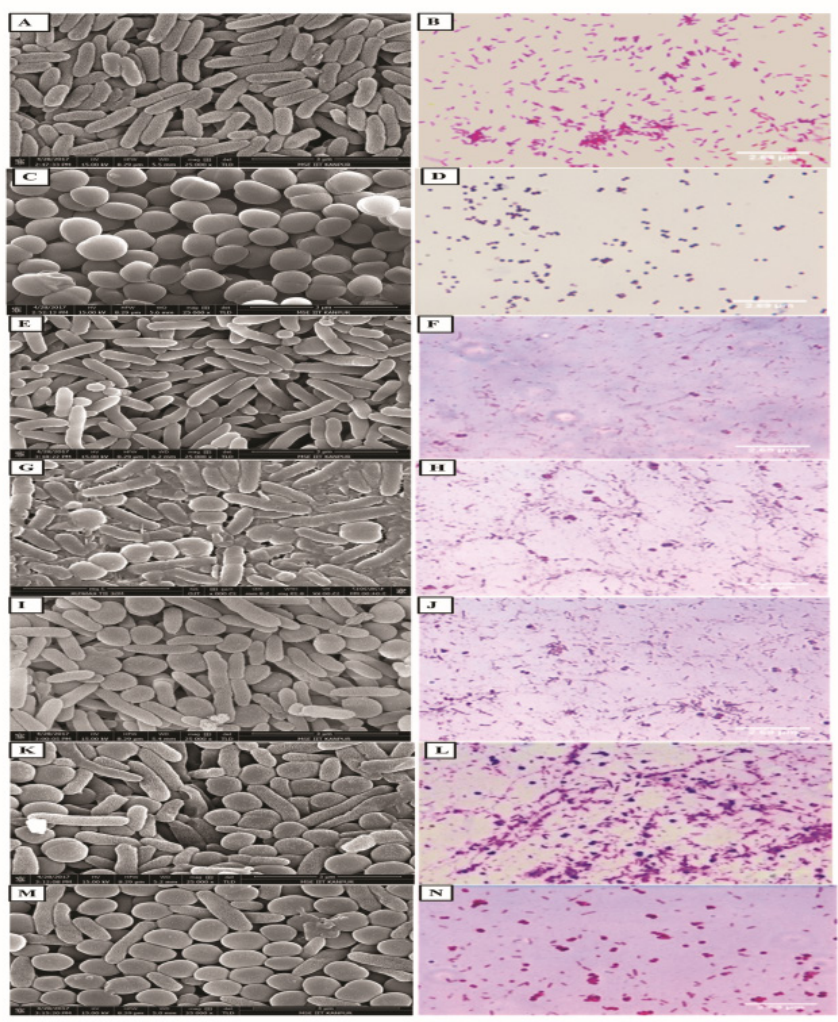

Figure 4: Scanning electron microscopy and light microscopy (Gram-staining) observation of $P$. aeruginosa (A and $\mathrm{B})$ and $S$. epidermidis $(\mathrm{C}$ and $\mathrm{D})$ alone and mixed cultures M1 (E and F), M2 (G and H), M3 (I and J), M4 (K and L), and M5 (M and N). Purple/blue, spherical shapes indicate $S$. epidermidis, pink rod shapes denote $P$. aeruginosa (Gram-staining B, D, F, H, J, L, and N), images captured at 100×. SEM images (A, C, E, G, I, K, and M) of planktonic mixed cultures with scale bars $(3 \mu \mathrm{m})$.

\subsubsection{Azocasein assay}

Pseudomonas aeruginosa-associated protease has a well-known function in ocular infections and sepsis, where they are able to interrupt epithelial tight junctions and break immunoglobulin and fibrin. Proteases are among the common virulence factor which helps in co-survival and pathogenicity. Total protease activity was examined by using skimmed milk agar plate assay, and it was observed that in comparison to $P$. aeruginosa alone, protease activity reduced significantly in M1-M3 mixed cultures (Figure 5B), which might be due to adaptation and competition for nutrient resources and persistence in a mixed culture environment [16].

\subsubsection{Alginate production}

Alginate is among the major components of exopolysaccharide produced by $P$. aeruginosa and contributes to biofilm matrix formation and bacterial colonization during infection and is thought to provide protection against antibiotic opsonophagocytosis. The alginate production was investigated, and its production was reduced, notably with different mixed cultures in comparison to PAO1 alone (Figure 5C). This finding was in contrast to the earlier investigation, which stated that the expression of virulence factors, especially alginate and alkaline protease gene, was upregulated in P. aeruginosa and S. maltophilia co-cultures [8]. 

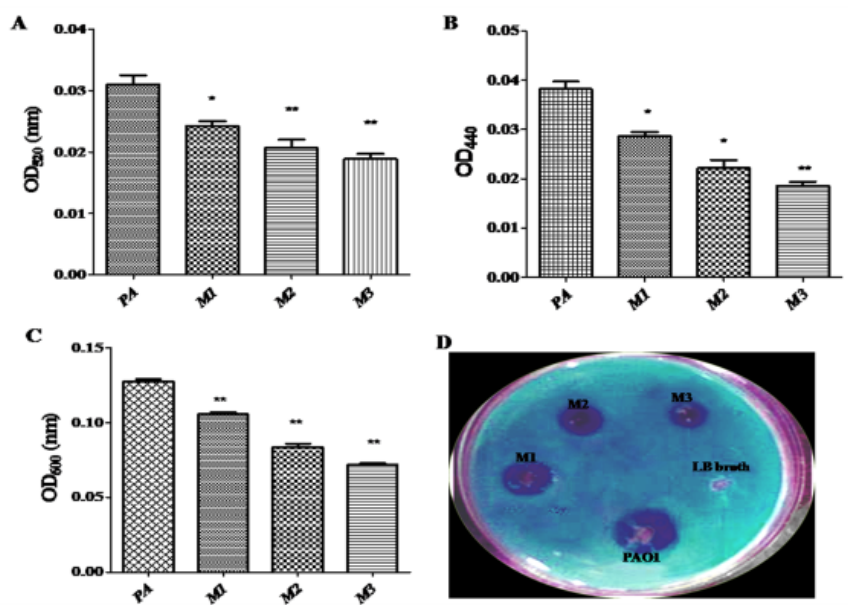

Figure 5: Virulence factors production by $P$. aeruginosa during interaction with $S$. epidermidis. (A) pyocyanin production, (B) protease activity (azocasein assay), (C) alginate production, (D) rhamnolipid production in mixed cultures (M1-M3). Error bars indicate the standard deviations for three measurements. ${ }^{*} p<0.05$ compared with the control. ${ }^{* *} p<0.001$ when compared with the control.
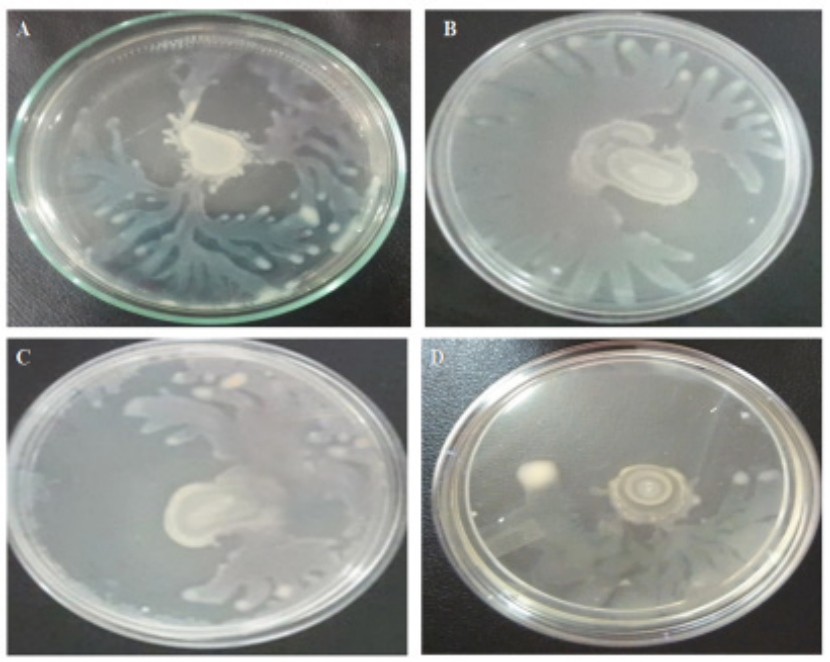

Figure 6: Swarming motility of $P$. aeruginosa alone and in mixed cultures. (A) $P$. aeruginosa alone and (B-D) M1-M3 mixed cultures.

\subsubsection{Rhamnolipid production}

Rhamnolipids are involved in the development of biofilm and also play an important role in surface-associated modes of bacterial motility. The rhamnolipids production was demonstrated by the dense blue zone around the wells. The production of rhamnolipids in mixed cultures was reduced in comparison to PAO1 alone. The diameter of the blue ring of rhamnolipids produced around the wells was measured. It was found to be $1.8 \pm 0.11,1.3 \pm 0.14,0.9$ \pm 0.10 , and $0.6 \pm 0.11 \mathrm{~cm}$ for $P$. aeruginosa alone, M1, M2, and M3 mixed cultures, respectively (Figure 5D).

\subsubsection{Swarming assay}

Swarming motility is widespread among flagellated bacteria and is a multicellular phenomenon involving the colonization and rapid

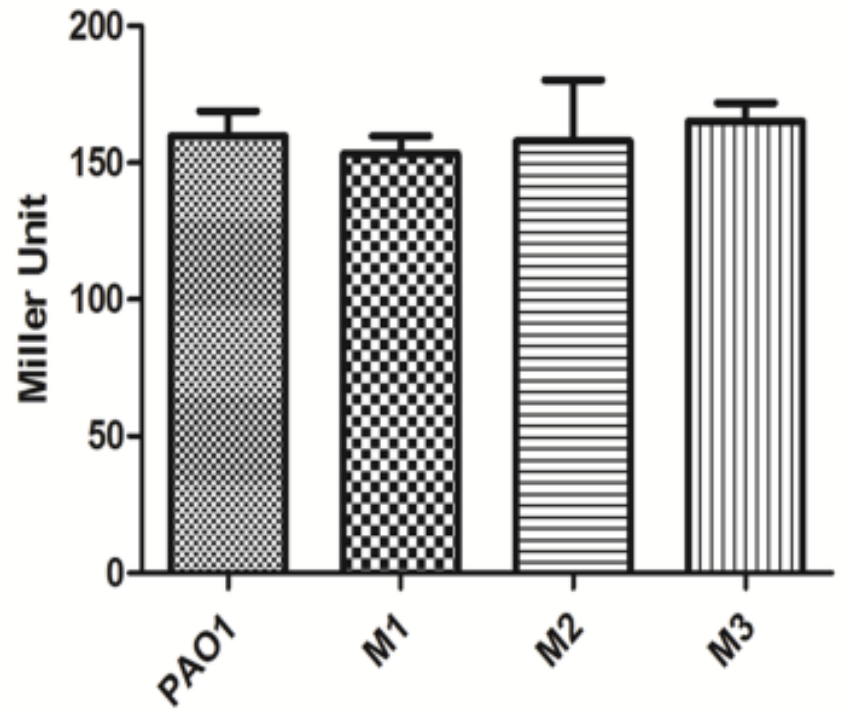

Figure 7: Detection of 3-oxo- $\mathrm{C}_{12}$ HSL level (long-chain QS molecules) produced by $P$. aeruginosa in mixed cultures supernatant with respect to control. Error bars indicate the \pm standard deviations of the measurements.

movement of a bacterial population across a semisolid medium. It is highly dependent on bacterial cell density, nutrients, and surface conditions. Swarming movement is distinguished by tendril formation (expanding irregular branching pattern) where microorganisms relocate from the point of inoculums to precisely defined medium, and this movement facilitates the transmission of $P$. aeruginosa infection. Swarming motility of $P$. aeruginosa alone and in mixed culture was tested on a $0.5 \%$ agar plate, and the length of tendrils in all the directions ( $x, y$, and $z$-axis) was measured. Swarming colony morphology of $P$. aeruginosa alone and in M1 mixed culture, expanding and irregular branching pattern was observed, but in the mixed cultures M2 and M3, swarming colony morphology of $P$. aeruginosa was affected; it remained localized to form expanding and irregular branching patterns in few directions only (Figure 6A-D). In mixed culture media, reduction in bud-like projections, expanding, and irregular branching pattern was observed as compared to control. This indicates the decrease in cell-to-cell contact, consequently reducing the spreading of infection. The findings showed that $S$. epidermidis presence could reduce $P$. aeruginosa-associated virulence factors and hence may effectively contribute to control infection, pathogenesis, and motility PAO1 [17].

\subsection{Detection of QS Molecules Using Bioreporter Strain}

It was reported that the virulence factors produced by $P$. aeruginosa are majorly regulated by the $\mathrm{C}_{12}$-HSL signal molecule [18]. Therefore, the production of $\mathrm{C}_{12}$ - $\mathrm{HSL}$ in the pure and mixed culture supernatants using bioreporter strain $E$. coli pJN105L pSC11 was studied. The expression of $P$. aeruginosa-associated virulence factors is majorly regulated by the QS systems. $\mathrm{C}_{12}$-HSL acts as a master regulator in the $P$. aeruginosa $\mathrm{AHL}-$ based $\mathrm{QS}$ pathway. The production of $\mathrm{C}_{12}$-HSL was studied in mixed cultures supernatant using bioreporter strain $E$. coli pJN105L pSC11. No significant change was observed in Miller units of mixed cultures M1-M3 in 
comparison to PAO1 alone. The results indicate that the production of $\mathrm{C}_{12}$-HSL in M1-M3 mixed cultures was similar to the PAO1 alone (Figure 7). This indicates that the production of $\mathrm{C}_{12}-\mathrm{HSL}$ in $P$. aeruginosa was not affected in the presence of $S$. epidermidis. Our findings are consistent with the finding of various researchers who reported quorum-independent expression/repression of $P$. aeruginosa-associated virulence factors [8]. Besides, earlier studies have shown that the Las system's repressor is a global regulator that controls various QS-regulated genes at the transcription level without affecting $\mathrm{C}_{12}$-HSL production $[19,20]$.

\section{CONCLUSION}

The study concludes that competitive interaction was found between P. aeruginosa and S. epidermidis. Pseudomonas aeruginosa inhibits the growth of $S$. epidermidis, while $S$. epidermidis reduced the virulence factors production of $P$. aeruginosa independent of $\mathrm{C}_{12}{ }^{-}$ HSL molecules production. The study demonstrated the significant role of $S$. epidermidis in reducing $P$. aeruginosa-associated infection and virulence factors for the first time.

\section{ACKNOWLEDGMENTS}

The authors are grateful to the Ministry of Human Resource Development (MHRD), Science and Engineering Research Board (SERB) (EMR/2014/000496), Department of Biotechnology (DBT) (BT/PR6175/GBD/27/367/2012), Ministry of Science and Technology, Government of India and TEQIP for their assistance and financial support. We are thankful to Prof. Peter Greenberg for the E. coli pJN105LpSC11 strain. We are also thankful to IIT Kanpur for providing the SEM facility.

\section{AUTHOR CONTRIBUTIONS}

All authors made substantial contributions to conception and design, acquisition of data, or analysis and interpretation of data; took part in drafting the article or revising it critically for important intellectual content; agreed to submit to the current journal; gave final approval of the version to be published; and agree to be accountable for all aspects of the work. All the authors are eligible to be an author as per the international committee of medical journal editors (ICMJE) requirements/guidelines.

\section{CONFLICT OF INTEREST}

The authors have declared that no conflict of interest exists.

\section{ETHICAL APPROVALS}

This study does not involve experiments on animals or human subjects.

\section{REFERENCES}

1. Grice EA, Segre JA. The skin microbiome. Nat Rev Microbiol 2011;9(4):244-53.

2. Pearson JP, Pesci EC, Iglewski BH. Roles of Pseudomonas aeruginosa las and rhl quorum-sensing systems in control of elastase and rhamnolipid biosynthesis genes. J Bacteriol 1997;179(18):5756-67.

3. Veesenmeyer JL, Hauser AR, Lisboa T, Rello J. Pseudomonas aeruginosa virulence and therapy: evolving translational strategies. Crit Care Med 2009;37(5):1777-86.
4. Qin Z, Yang L, Qu D, Molin S, Tolker-Nielsen T. Pseudomonas aeruginosa extracellular products inhibit staphylococcal growth, and disrupt established biofilms produced by Staphylococcus epidermidis. Microbiology (Reading) 2009;155(7):2148-56.

5. Fugère A, Séguin DL, Mitchell G, Déziel E, Dekimpe V, Cantin $\mathrm{AM}$, et al. Interspecific small molecule interactions between clinical isolates of Pseudomonas aeruginosa and Staphylococcus aureus from adult cystic fibrosis patients. PLoS One 2014;9(1):e86705.

6. Kalia M, Singh D, Sharma D, Narvi SS, Agarwal V. Senna alexandriana mill as a potential inhibitor for quorum sensing-controlled virulence factors and biofilm formation in Pseudomonas aeruginosa PAO1. Pharmacogn Mag 2020;16:797-802.

7. Kuznetsova MV, Maslennikova IL, Karpunina TI, Nesterova LY, Demakov VA. Interactions of Pseudomonas aeruginosa in predominant biofilm or planktonic forms of existence in mixed culture with Escherichia coli in vitro. Can J Microbiol 2013;59(9):604-10.

8. Pompilio A, Crocetta V, De Nicola S, Verginelli F, Fiscarelli E, Bonaventura GD. Cooperative pathogenicity in cystic fibrosis: Stenotrophomonas maltophilia modulates Pseudomonas aeruginosa virulence in mixed biofilm. Front Microbiol 2015;6:951.

9. Chu YY, Nega M, Wölfle M, Plener L, Grond S, Jung K, Götz F. A new class of quorum quenching molecules from Staphylococcus species affects communication and growth of Gram-negative bacteria. PLOS Pathog 2013;9(9):e1003654.

10. Fischer ER, Hansen BT, Nair V, Hoyt FH, Dorward DW. Scan electron microsc. Curr Protoc Microbiol 2012;25:2B.2.1-47.

11. Zheng H, Korendovych IV, Luk YY. Quantification of alginate by aggregation induced by calcium ions and fluorescent polycations. Anal Biochem 2016;492:76-81.

12. Vazquez SC, Rios Merino LN, MacCormack WP, Fraile ER. Proteaseproducing psychrotrophic bacteria isolated from Antarctica. Polar Biol 1995;15(2):131-5.

13. Siegmund I, Wagner F. New method for detecting rhamnolipids excreted by Pseudomonas species during growth on mineral agar. Biotechnol Tech 1991;5(4):265-8.

14. Baldan R, Cigana C, Testa F, Bianconi I, De Simone M, Pellin D, et al. Adaptation of Pseudomonas aeruginosa in cystic fibrosis airways influences virulence of Staphylococcus aureus in vitro and murine models of co-infection. PLoS One 2014;9(3):e89614.

15. Miller JH. Experiments in molecular genetics. Cold Spring Harbor Laboratory Press, Cold Spring Harbor, NY, vol 433, pp 352-5, 1972.

16. Hoge R, Pelzer A, Rosenau F, Wilhelm S. Weapons of a pathogen: proteases and their role in virulence of Pseudomonas aeruginosa. Curr Res Technol Educ Top Appl Microbiol Microb Biotechnol 2010;2:383-95.

17. Venturi V, Bertani I, Kerényi A, Netotea S, Pongor S. Co-swarming and local collapse: quorum sensing conveys resilience to bacterial communities by localizing cheater mutants in Pseudomonas aeruginosa. PLoS One 2010;5(4):e9998.

18. Rumbaugh KP. Genomic complexity and plasticity ensure Pseudomonas success. FEMS Microbiol Lett 2014;356(2):141-3.

19. Lee J, Wu J, Deng Y, Wang J, Wang C, Wang J, et al. A cell-cell communication signal integrates quorum sensing and stress response. Nat Chem Biol 2013;9(5):339-43.

20. Reis RS, Pereira AG, Neves BC, Freire DMG. Gene regulation of rhamnolipid production in Pseudomonas aeruginosa-a review. Bioresour Technol 2011;102(11):6377-84.

How to cite this article:

Dohare S, Singh D, Sharma D, Agarwal V. The effect of Staphylococcus epidermidis cells on Pseudomonas aeruginosa-associated virulence factors. J Appl Biol Biotech 2021; 9(06):122-127. 